\title{
A GAP THEOREM
}

\author{
BY \\ M. $\operatorname{KAC}^{(1)}$, R. SALEM AND A. ZYGMUND
}

1. Introduction. Let $f(x)$ be periodic, with period 1 , and belong to the class $L$. Let $a$ be a positive integer greater than 1 . Raïkov proved( $\left.{ }^{2}\right)$ that for almost all $x$

$$
\lim _{m \rightarrow \infty} \frac{f(x)+f(a x)+\cdots+f\left(a^{m} x\right)}{m+1}=\int_{0}^{1} f(x) d x .
$$

As F. Riesz pointed out $\left({ }^{3}\right)$ this theorem is actually an instance of the classical ergodic theorem and can be proved by exactly the same methods as the ergodic theorem. The present paper has its origin in the question as to whether (1) remains true when $a$ is not an integer, but any number larger than one $\left({ }^{4}\right)$. We are unable to answer this question in the general case when $f \in L$, but under stronger assumptions we shall prove the following stronger result:

THEOREM. Let $f(x)$ be periodic (with period $2 \pi$, to fix the ideas) and belong to the class $L^{2}$, and let its mean-value be zero. Suppose, in addition, that

$$
\int_{0}^{2 \pi}\left(f-s_{n}\right)^{2} d x=O(\log n)^{-\sigma}
$$

where $\sigma>0$ and $s_{n}$ denotes the nth partial sum of the Fourier series of $f$. Let $\left\{\lambda_{k}\right\}$ be any positive sequence such that $\lambda_{k+1} / \lambda_{k} \geqq q>1$. Then:

(1) If $\sigma>2$, the series $\sum c_{k} f\left(\lambda_{k} x\right)$ converges for almost all $x$, provided $\sum c_{k}^{2} \log ^{2} k<\infty$.

(2) If $\sigma \leqq 2$ the series $\sum f\left(\lambda_{k} x\right) / k^{1-\delta}$ converges for almost all $x$ for every $\delta<\sigma / 4$.

The result that, for almost all $x$,

$$
\lim _{m \rightarrow \infty} \frac{f(x)+f\left(\lambda_{1} x\right)+\cdots+f\left(\lambda_{m} x\right)}{m+1}=0
$$

under the conditions stated is a weak consequence of the theorem, since it

Presented to the Society, April 26, 1947; received by the editors February 1S, 1947.

(1) John Simon Guggenheim Memorial Fellow.

(2) Rec. Math. (Mat. Sbornik) N.S. vol. 1 (1936) pp. 377-383.

(3) Comment. Math. Helv. vol. 17 (1945) p. 221.

(4) The answer is known, of course, when $f$ is bounded and integrable in the Riemann sense, since the fractional part of $q^{m} x(q>1)$ is uniformly distributed for almost all $x$. 
follows from the fact that the series $\sum k^{-1} f\left(\lambda_{k} x\right)$ converges almost everywhere.

2. Remarks on quasi-orthogonal functions. Let $f_{n}(x), n=1,2, \cdots$, be any sequence of functions (which we assume real only to simplify the exposition') all belonging to $L^{2}$ in $(a, b)$. Let $a_{j k}=\int_{a}^{b} f_{j} f_{k} d x$. Let us suppose that the quadratic form $\sum \sum a_{j k} x_{j} x_{k}$ is bounded in Hilbert space, with bound $M$. Let $F(x) \in L^{2}$ in $(a, b)$ and let $c_{n}=\int_{a}^{b} F f_{n} d x$. Then $\sum_{1}^{\infty} c_{n}^{2} \leqq M \int_{a}^{b} F^{2} d x$ or, in other words, Bessel's inequality holds except for the introduction of the constant $M$. This remark is due to Boas $\left.{ }^{5}\right)$ who considered also spaces other than Hilbert spaces. In our case, the proof is immediate by writing

$$
\begin{aligned}
0 & \leqq \int_{a}^{b}\left(F-\gamma_{1} f_{1}-\cdots-\gamma_{n} f_{n}\right)^{2} d x=\int_{a}^{b} F^{2} d x-2 \sum_{1}^{n} \gamma_{k} c_{k}+\sum_{1}^{n} \sum a_{j k} \gamma_{j} \gamma_{k} \\
& \leqq \int_{a}^{b} F^{2} d x-2 \sum_{1}^{n} \gamma_{k} c_{k}+M \sum_{1}^{n} \gamma_{k}^{2} \\
& =\int_{a}^{b} F^{2} d x+\sum_{1}^{n}\left(M^{1 / 2} \gamma_{k}-\frac{c_{k}}{M^{1 / 2}}\right)^{2}-\frac{1}{M} \sum_{1}^{n} c_{k}^{2}
\end{aligned}
$$

and taking $\gamma_{k}=c_{k} / M$. A system $\left\{f_{n}\right\}$ such that the quadratic form $\sum \sum a_{j k} x_{j} x_{k}$ is bounded will be called "quasi-orthogonal." The name has already been used by R. Bellman $\left(^{6}\right)$ under somewhat stronger hypotheses.

Let us now point out a few simple consequences of quasi-orthogonality, which do not seem to have been stated. The most important one which we shall use here is that every theorem on orthogonal systems whose proof depends only on Bessel's inequality holds for quasi-orthogonal systems: in particular, Menchoff's theorem, asserting the convergence almost everywhere of $\sum c_{n} f_{n}(x)$ provided $\sum c_{n}^{2} \log ^{2} n<\infty$, holds.

Let us also observe that, given any sequence $\left\{f_{n}\right\}$ of functions belonging to $L^{2}$ in $(a, b)$, the necessary and sufficient condition in order that it be quasiorthogonal is that $\sum c_{n} f_{n}$ should converge in the mean, with exponent 2 , for every sequence $\left\{c_{n}\right\}$ with $\sum c_{n}^{2}<\infty$. The necessity of the condition follows immediately from Bessel's inequality, and the sufficiency from the fact that under the hypothesis of convergence in the mean the integral $\int_{a}^{b}\left(\sum_{1}^{n} c_{k} f_{k}\right)^{2} d x$ is bounded for every sequence $\left\{c_{n}\right\}$ with $\sum c_{n}^{2}<\infty$.

Using this remark, we can state the above extension of Menchoff's theorem in the following way: whenever the sequence of functions $f_{n} \in L^{2}$ in $(a, b)$ is such that $\sum c_{n} f_{n}$ converges in the mean with exponent 2 for all sequences $\left\{c_{n}\right\}$ with $\sum c_{n}^{2}<\infty$, then also the series $\sum c_{n} f_{n}$ converges almost everywhere whenever $\sum c_{n}^{2} \log ^{2} n<\infty$.

We make a last remark which we shall not use here, that is, that the

(5) Amer. J. Math. vol. 63 (1941) p. 361.

(8) Bull. Amer. Math. Soc. vol. 50 (1944) p. 517. 
necessary and sufficient condition for quasi-orthogonality can be given in a different form if we assume that each $f_{k}$ is given in $(a, b)$ by its Fourier expansion in terms of a system $\left\{\phi_{n}\right\}$ orthonormal and complete in $(a, b)$; $f_{k} \sim \sum_{n=1}^{\infty} b_{k n} \phi_{n}$. Then $a_{j k}=\sum_{n} b_{j n} b_{k n}$, and one sees that the quadratic form $\sum \sum a_{j k} x_{j} x_{k}$ is the norm of the bilinear form $\sum \sum b_{j k} x_{j} y_{k}$. Thus, the boundedness of this form is necessary and sufficient for the quasi-orthogonality of the system $\left\{f_{n}\right\}$. This shows also that the system of functions $g_{k} \sim \sum_{1}^{\infty} b_{n k} \phi_{n}$ is quasi-orthogonal if and only if the system $\left\{f_{k}\right\}$ is so.

We now state the lemma of which we shall make use.

LemMa I. Let $f_{n}(x), n=1,2, \cdots$, be any sequence of functions all belonging to $L^{2}$ in $(a, b)$. Suppose that for all $j \neq k$

$$
\left|\int_{a}^{b} f_{j}(x) f_{k}(x) d x\right| \leqq \frac{C}{|j-k|^{e}}
$$

and that $\int_{a}^{b} f_{k}^{2}(x) d x \leqq C^{\prime}$. Then:

(1) If $\epsilon>1$, the series $\sum c_{n} f_{n}(x)$ converges almost everywhere in $(a, b)$ provided $\sum c_{n}^{2} \log ^{2} n<\infty$.

(2) If $\epsilon \leqq 1$ the series $\sum f_{n}(x) / n^{1-\delta}$ converges almost everywhere in $(a, b)$ for every $\delta<\epsilon / 2$.

The first part of the lemma follows immediately from the fact that, under the condition (4), the system $\left\{f_{n}(x)\right\}$ is quasi-orthogonal in $(a, b)$ if $\epsilon>1$.

To prove the second part, suppose first $\epsilon<1$. and remark that by a result of Hardy, Littlewood, and Pólya $\left({ }^{7}\right)$ the bilinear form

$$
\sum_{j \neq k} \sum_{k} \frac{x_{j} y_{k}}{j^{(1-\epsilon) / 2} k^{(1-\epsilon) / 2}|j-k|_{\epsilon}^{\epsilon}}, \quad 0<\epsilon<1,
$$

is bounded in Hilbert space. Hence the condition (4) implies that the system $\left\{f_{n}(x) / n^{(1-\epsilon) / 2}\right\}$ is quasi-orthogonal, and the series $\sum c_{n} f_{n}(x) / n^{(1-\epsilon) / 2}$ converges almost everywhere whenever $\sum c_{n}^{2} \log ^{2} n<\infty$. Taking $c_{n}=n^{-\theta}$ with any $\theta>1 / 2$ we have the second part of our lemma for $\epsilon<1$ and it is seen immediately that it holds also for $\epsilon=1$.

3. Lemmas on bilinear forms. Let us consider the two bilinear forms

$$
A(\alpha)=\sum_{i, j=1}^{\infty} \sum_{\frac{1}{\alpha}+j} \frac{x_{i} y_{j}}{\alpha i+j}, \quad B(\alpha)=\sum_{i, j=1}^{\infty} \sum^{\prime} \frac{x_{i} y_{i}}{\alpha i-j}
$$

where $\alpha$ is real and not less than 1 , the prime in the second form meaning that terms for which $|\alpha i-j|<1$ are omitted. We are specially concerned here with the behavior of these forms as $\alpha \rightarrow \infty$.

(7) See Inequalities, p. 227. 
Lemma II. The bound of $A(\alpha)$ in Hilbert space does not exceed $C_{1} / \alpha^{1 / 2}$ where $C_{1}$ is an absolute constant, that is,

$$
|A(\alpha)| \leqq \frac{C_{1}}{\alpha^{1 / 2}}\left(\sum_{1}^{\infty}\left|x_{i}\right|^{2}\right)^{1 / 2}\left(\sum_{1}^{\infty}\left|y_{i}\right|^{2}\right)^{1 / 2} .
$$

LEMma III. The bound of $B(\alpha)$ in Hilbert space does not exceed an absolute constant $C_{2}$,

$$
|B(\alpha)| \leqq C_{2}\left(\sum_{1}^{\infty}\left|x_{i}\right|^{2}\right)^{1 / 2}\left(\sum_{1}^{\infty}\left|y_{j}\right|^{2}\right)^{1 / 2} .
$$

Lemma IV. Denoting by $\rho(\nu)$ the ratio

$$
\left(\sum_{[\nu]+1}^{\infty}\left|y_{j}\right|^{2}\right)^{1 / 2} /\left(\sum_{1}^{\infty}\left|y_{j}\right|^{2}\right)^{1 / 2}
$$

one has

$$
|B(\alpha)| \leqq C_{3}\left(\frac{1}{\alpha^{1 / 2}}+\rho\left(\frac{\alpha}{2}\right)\right)\left(\sum_{1}^{\infty}\left|x_{i}\right|^{2}\right)^{1 / 2}\left(\sum_{1}^{\infty}\left|y_{j}\right|^{2}\right)^{1 / 2},
$$

$C_{8}$ being an absolute constant.

Lemma II is well known with $C_{1}=\pi$, as an immediate consequence of the Hardy, Littlewood, and Pólya extension of Hilbert's first bilinear form $\left({ }^{8}\right)$.

In order to prove Lemma III, let us suppose first $1 \leqq \alpha \leqq 2$. We consider two functions $f(x)$ and $g(x)$ belonging to $L^{2}$ in $(-\infty,+\infty)$ and write the equation:

$$
J=\int_{-\infty}^{+\infty} \int_{-\infty}^{+\infty} \frac{f(x) g(y)}{\alpha x-y} d x d y=\frac{1}{\alpha} \int_{-\infty}^{+\infty} \int_{-\infty}^{+\infty} \frac{f(x / \alpha) g(y)}{x-y} d x d y,
$$

the first integral being extended to the domain $|\alpha x-y| \geqq 1$ and the second one to the domain $|x-y| \geqq 1$. Considering the second integral we remark that by M. Riesz's well known theorem $\left(^{9}\right)$

$$
\begin{aligned}
|J| & <\frac{C}{\alpha}\left(\int_{-\infty}^{+\infty}\left|f\left(\frac{x}{\alpha}\right)\right|^{2} d x\right)^{1 / 2}\left(\int_{-\infty}^{\infty}|g(y)|^{2} d y\right)^{1 / 2} \\
& =\frac{C}{\alpha^{1 / 2}}\left(\int_{-\infty}^{+\infty}|f(x)|^{2} d x\right)^{1 / 2}\left(\int_{-\infty}^{\infty}|g(y)|^{2} d y\right)^{1 / 2}
\end{aligned}
$$

$C$ being an absolute constant. We take now for $f$ and $g$ step functions $f=0$

(8) See Inequalities, p. 227.

(?) M. Riesz, Sur les fonctions conjuguées. Math. Zeit. vol. 27 (1927) pp. 218-244, especially pp. 240-241. 
for $x<1, f=x_{i}$ for $i \leqq x<i+1(i=1,2, \cdots), g=0$ for $y<1, g=y_{i}$ for $j \leqq y<j$ $+1(j=1,2, \cdots)$; the integral $J$ becomes a bilinear form $\sum \sum b_{i j} x_{i} y_{i}$. For all terms for which, say, $|\alpha i-j|>2(\alpha+1)$ it is immediately seen that

$$
\left|b_{i j}-\frac{1}{\alpha i-j}\right|<\frac{C^{\prime} \alpha}{(\alpha i-j)^{2}},
$$

$C^{\prime}$ being an absolute constant. Hence the inequality (6) gives immediately (5) for $1 \leqq \alpha \leqq 2$. For general values of $\alpha$ take $\alpha=m \alpha_{0}, m$ being an integer and $1 \leqq \alpha_{0} \leqq 2$. Then

$$
\sum \sum^{\prime} \frac{x_{i} y_{j}}{m \alpha_{0} i-j}=\sum \sum^{\prime} \frac{\xi_{k} y_{j}}{\alpha_{0} k-j},
$$

where $\xi_{k}=x_{i}$ for $k=m i$ and $\xi_{k}=0$ otherwise. This proves (5) for all $\alpha \geqq 1$.

In order to prove Lemma IV write

$$
B(\alpha)=\sum \sum^{\prime} \frac{x_{i} y_{j}}{\alpha i-j}=\sum_{j \leqq \alpha / 2} \sum^{\prime}+\sum_{j>\alpha / 2} \sum^{\prime}=B_{1}+B_{2},
$$

say. In $B_{1}, \alpha i-j \geqq(\alpha i+j) / 3$, hence, by Lemma II, $B_{1}$ has bound $3 C_{1} / \alpha^{1 / 2}$. In $B_{2}$ we apply Lemma III and we get

$$
\begin{aligned}
\left|B_{2}\right| \leqq & C_{2}\left(\sum_{1}^{\infty}\left|x_{i}\right|^{2}\right)^{1 / 2}\left(\sum_{[\alpha / 2]+1}^{\infty}\left|y_{j}\right|^{2}\right)^{1 / 2} \\
& =C_{2}\left(\sum_{1}^{\infty}\left|x_{i}\right|^{2}\right)^{1 / 2}\left(\sum_{1}^{\infty}\left|y_{j}\right|^{2}\right)^{1 / 2} \rho\left(\frac{\alpha}{2}\right)
\end{aligned}
$$

and, collecting the results, Lemma IV follows.

4. Proof of the theorem. We may obviously suppose $\lambda_{1} \geqq 1$. Let $f(x)$ belong to $L^{2}$, have period $2 \pi$, and mean value zero. Let

$$
f(x) \sim \sum_{-\infty}^{\infty} c_{n} e^{i n x} \quad\left(c_{0}=0, c_{-n}=\bar{c}_{n}\right)
$$

be its Fourier expansion. Let

$$
a_{j k}=\int_{a}^{b} f\left(\lambda_{j} x\right) f\left(\lambda_{k} x\right) d x, \quad \lambda_{k}>\lambda_{i} \geqq 1 .
$$

We have

$$
a_{j k}=\frac{1}{\lambda_{j}} \int_{a \lambda_{j}}^{b \lambda_{j}} f(x) f(\alpha x) d x, \quad \alpha=\frac{\lambda_{k}}{\lambda_{j}}>1 .
$$

If we denote now by $M(\alpha)$ and $N(\alpha)$ the respective bounds in Hilbert space for the forms $A(\alpha)$ and $B(\alpha)$, considered above, we see that 


$$
\left|a_{j k}\right| \leqq \frac{4}{\lambda_{j}}[M(\alpha)+N(\alpha)] \sum_{1}^{\infty}\left|c_{n}\right|^{2}+2(b-a) \sum^{*}\left|c_{p} c_{q}\right|,
$$

the starred sum being extended to the integers $p, q \geqq 1$ such that $|\alpha p-q|<1$.

We write now

$$
\sum_{[\nu]+1}^{\infty}\left|c_{n}\right|^{2}=R(\nu)
$$

and we remark that since in the starred sum $q>\alpha-1$, the last term in (7) is less than $C(R(\alpha / 2))^{1 / 2}, C$ being a constant depending on $f(x)$ and on $(a, b)$ but not on $j$ and $k$. Using the bounds given by Lemma II and Lemma IV, we deduce that

$$
\left|a_{j k}\right| \leqq \frac{D}{\alpha^{1 / 2}}+E\left(R\left(\frac{\alpha}{2}\right)\right)^{1 / 2},
$$

$D$ and $E$ being constants depending on $f(x), a, b$, but not on $j, k$. If we use now the hypothesis (2) of our theorem, we can write

$$
\left|a_{j k}\right|<\frac{A}{|j-k|^{\sigma / 2}},
$$

$A$ being independent of $j, k$. The appraisal of $a_{j j}$ presents no difficulty and the two parts of our theorem follow now immediately from this inequality and from Lemma I.

5. Application. As an application, consider the infinite product $\gamma(u)$ $=\prod_{k=0}^{\infty} \cos \pi u \xi^{k}(0<\xi<1)$ which is the characteristic function of the well known distribution function obtained by infinitely many convolutions of a Bernouilli distribution function. Let $\xi=1 / \theta, \theta>1$, and let $u=\lambda \theta^{m}(1 / \theta<\lambda \leqq 1)$. Then

$$
\gamma\left(\lambda \theta^{m}\right)=\cos \pi \lambda \theta^{m} \cdot \cos \pi \lambda \theta^{m-1} \cdots \cos \pi \frac{\lambda}{\theta} \cdot \cos \pi \frac{\lambda}{\theta^{2}} \cdots
$$

Excepting special values of $\lambda$ for which $\gamma\left(\lambda \theta^{m}\right)=0$, we see that

$$
\gamma\left(\lambda \theta^{m}\right)=A(\lambda) \cdot \cos \pi \lambda \cos \pi \lambda \theta \cdots \cos \pi \lambda \theta^{m}=A(\lambda) \cdot P,
$$

where $A(\lambda) \neq 0$ is independent of $m$. Now, the function $\log \cos ^{2} \pi x$ has period 1 , and Fourier coefficients $O(1 / n)$. Hence by our theorem

$$
\frac{\log \cos ^{2} \pi \lambda+\cdots+\log \cos ^{2} \pi \lambda \theta^{m}}{m+1} \rightarrow \int_{0}^{1} \log \cos ^{2} \pi x d x=-2 \log 2
$$

almost everywhere in $\lambda$, that is to say, for almost all $\lambda,\left(P^{2}\right)^{1 / m} \rightarrow 1 / 4$. One has, for such a $\lambda$, 


$$
\frac{1}{A(\lambda)} \cdot \frac{1}{(2+\epsilon)^{m}}<\left|\gamma\left(\lambda \theta^{m}\right)\right|<\frac{1}{A(\lambda)} \frac{1}{(2-\epsilon)^{m}}
$$

for $\epsilon$ arbitrarily small, as $m \rightarrow \infty$. Thus, for infinitely many values of $u$

$$
\frac{C}{u^{\alpha+\eta}}<|\gamma(u)|<\frac{C}{u^{\alpha-\eta}}
$$

where $C$ is a constant, $\eta$ is arbitrarily small, and $\alpha$ denotes the number $\log 2 / \log \dot{\theta}$.

6. Other applications of quasi-orthogonality. The notion of quasiorthogonality is applicable to a strong theorem of the ergodic type. Let $x$ denote a point in a finite-dimensional Euclidean space $R$, let $f(x) \in L^{2}$ in a set $S$ of finite measure in $R$, and let $T$ be a transformation such that $T x \in S$ when $x \in S$. We suppose that $T$ is measure preserving in the wide sense, that is, if $\varepsilon$ is the set of all points $x$ such that $T x \in E$, then $|\varepsilon|=|E|$. We assume, in addition, that if $I$ and $J$ are two rectangular cells in $S$ and if $J_{-m}$ is the set of all $x$ such that $T^{m} x \in J$, then

$$
\left|I \cdot J_{-m}\right| \rightarrow|I| \cdot|J|
$$

as $m \rightarrow \infty$ (strong mixing). It is then an easy and known consequence that if $f$ has mean value zero in $S$,

$$
\delta(m)=\int_{S} f(x) f\left(T^{m} x\right) d v \rightarrow 0 \quad \text { as } m \rightarrow \infty .
$$

Suppose now that, for a given $f, \delta(m)$ is of order $m^{-\epsilon}(\epsilon>0)$; then if $k>j$

$$
\int_{S} f\left(T^{i} x\right) f\left(T^{k} x\right) d v=\int_{S} f(x) f\left(T^{k-j} x\right) d v=O\left\{(k-j)^{-\bullet}\right\}
$$

and we can apply the first or the second result of Lemma I, according as $\epsilon$ is greater than 1 or not greater than 1 . In both cases we get a stronger result than the classical ergodic theorem.

Another application of quasi-orthogonality is the following one.

In a paper of $\mathrm{R}$. Fortet $\left({ }^{10}\right)$ one finds the following theorem, stated without proof:

"Pour une suite discrete de variables aléatoires $x_{n}$ telles que

$$
E\left(x_{n}^{2}\right)=1, \quad E\left(x_{n} x_{n+u}\right)=R(u)
$$

s'il existe un nombre positif $\alpha$ tel que, pour $u$ assez grand, on ait $|R(u)|<u^{-\alpha}$, la moyenne

(10) Sur une suite également repartie, Studia Mathematica vol. 9 (1940) pp. 54-69 and particularly p. 57. 


$$
\frac{1}{n} \sum_{i=1}^{n} x_{i}
$$

tend presque surement vers zero( $\left.{ }^{11}\right)$. Et tres vraisemblablement ce résultat subsiste pour une suite continue."

From our point of view, the result for the discrete case is immediate, even in a stronger form. For if $\left\{x_{n}(\omega)\right\}$ is a sequence of $L^{2}$ functions in some space $\boldsymbol{\Omega}$ in which a Lebesgue measure has been defined, and if

$$
\int_{\Omega} x_{n}(\omega) d \mu=0, \quad \int_{\Omega} x_{n}^{2}(\omega) d \mu=1, \quad \int_{\Omega} x_{n}(\omega) x_{n+u}(\omega) d \mu=O\left(u^{-\alpha}\right),
$$

we can apply the results of Lemma I.

In the continuous case, one has a one-parameter family of functions $x_{t}(\omega)$ belonging to $L^{2}$ in $\Omega$ for every $t$, and such that

$$
\int_{\Omega} x_{t}(\omega) d \mu=0, \quad \int_{\Omega} x_{t}^{2}(\omega) d \mu=1, \quad \int_{\Omega} x_{t}(\omega) x_{t+u}(\omega) d \mu=R(u)=O\left(u^{-\alpha}\right),
$$

and we want to prove that

$$
\lim _{T \rightarrow \infty} \frac{1}{T} \int_{0}^{T} x_{t}(\omega) d t=0
$$

almost everywhere in $\omega$. We have, of course, to make certain assumptions about the integrability of $x_{t}(\omega)$ as a function of $t$. We shall assume that, for almost all $\omega, x_{t}(\omega)$ is a function of $t$ belonging to $L^{2}$ in every finite interval.

Let us assume first that $T$ is an integer, $T=m$, and let

$$
f_{k}(\omega)=\int_{k-1}^{k} x_{t}(\omega) d t, \quad k=1,2,3, \cdots
$$

It is immediately seen, by application of Schwarz's inequality and Fubini's theorem, that $f_{k}(\omega) \in L^{2}$ in $\Omega$ and that

$$
\int_{\Omega} f_{j}(\omega) f_{k}(\omega) d \mu=\int_{j-1}^{j} \int_{k-1}^{k} R(|s-t|) d s d t<\frac{C}{|j-k|^{\alpha}},
$$

where $C$ is a constant, for, say, $|j-k|>4$.

This allows immediately the application of the results of Lemma I to the sequence $\left\{f_{k}(\omega)\right\}$ and proves Fortet's conjecture for $T$ an integer. To pass to the general case, we have only to show that, for almost all $\omega$,

$$
\frac{1}{T} \int_{T}^{T+1}\left|x_{t}(\omega)\right| d t=o(1) \quad \text { as } T \rightarrow \infty .
$$

(11) The random variables are supposed to have mean value zero. 
Let

$$
g_{n}(\omega)=\int_{n}^{n+2} x_{t}^{2}(\omega) d t
$$

We have, by Fubini's theorem,

$$
\int_{\Omega} \dot{g}_{n}(\omega) d \mu=\int_{n}^{n+2} d t \int_{\Omega} x_{t}^{2}(\omega) d \mu=2 .
$$

Hence, the series $\sum\left(1 / n^{2}\right) g_{n}(\omega)$ converges except in a set of measure zero Hence if $\omega$ does not belong to that exceptional set, $g_{n}(\omega)=o\left(n^{2}\right)$ and

$$
\int_{T}^{T+1}\left|x_{t}(\omega)\right| d t \leqq \int_{[T]}^{[T]+2}\left|x_{t}(\omega)\right| d t \leqq 2^{1 / 2}\left(g_{[T]}(\omega)\right)^{1 / 2}=o(T),
$$

which proves (8) and completes the proof of Fortet's conjecture.

In general, the use of quasi-orthogonal functions as stated in Lemma I gives simple but convenient forms of laws of large numbers for so-called stationary processes of order 2 . In many physical applications the correlation coefficient $R(u)$ tends to zero sufficiently fast to allow the application of the results stated in Lemma $I$.

CORNELl University, ITHACA, N. Y.

Massachusetts Institute of Technology, Cambridge, Mass.

University of Pennsylvania, Philadelphia, Pa. 